\title{
Evaluation of resistance to pyrethroid and organophosphate adulticides, and $k d r$ genotyping in Ades aegypti populations from Roraima, the Northernmost Brazilian State
}

\author{
Ramão Luciano Nogueira Hayd \\ Universidade Federal de Roraima \\ Luana Carrara \\ Fundacao Oswaldo Cruz \\ Joel de Melo Lima \\ Secretaria de Estado de Saúde de Roraima \\ Nathalia Coelho Vargas de Almeida \\ Secretaria de Estado da Saude de Roraima \\ José Bento Pereira Lima
}

Fundacao Oswaldo Cruz

Ademir Jesus Martins ( $\sim$ ademirji@ioc.fiocruz.br)

Fundacao Oswaldo Cruz https://orcid.org/0000-0001-5739-1215

\section{Research}

Keywords: Vector control, Insecticide Resistance Monitoring, Rorainópolis, Pacaraima, Bonfım, Boa vista, deltamethrin, malathion

Posted Date: January 20th, 2020

DOl: https://doi.org/10.21203/rs.2.21242/v1

License: (c) (1) This work is licensed under a Creative Commons Attribution 4.0 International License.

Read Full License

Version of Record: A version of this preprint was published at Parasites \& Vectors on May 20th, 2020. See the published version at https://doi.org/10.1186/s13071-020-04127-w. 


\section{Abstract}

Background Roraima is the northernmost State in Brazil and makes international borders with Venezuela and Guyana. Although mostly covered by the tropical forests, the urban centers are highly infested with Ae. aegypti and endemic for dengue, Zika and chikungunya. Here we presented the levels of Ae. aegypti infestation and number of arboviruses cases between 2015 and 2018 in the studied localities. We evaluated the insecticide resistance status of Ae. aegypti populations from the capital Boa Vista, two cities in international borders: Pacaraima and Bonfım, and Rorainópolis in the limit with Amazonas State, collected in 2016 and 2018.

Methods WHO like tube tests with the pyrethroid deltamethrin $(0.05$ and $0.12 \%)$ and the organophosphate malathion $(0.7 \%)$ were conducted. Genotyping of kdr mutations, related to resistance to pyrethroids, were performed for the SNP variations in the sites 1016 and 1534 of the voltage gated sodium channel gene (Na V) with a TaqMan qPCR approach.

Results Only Ae. aegypti was present in our collection, meaning that there is still a barrier that Ae. albopictus was still not able to transpose from Amazonas toward Roraima. All Ae. aegypti populations were susceptible to $0.7 \%$ malathion in 2016 , however mortality dropped to under $90 \%$ in Bonfim and Pacaraima in 2018. All populations were resistant to $0.5 \%$ deltamethrin in both years. The time that $50 \%$ of females suffered knockdown ( Kd T 50 ) under exposition to 0.5\% deltamethrin was 3.3-5.9 fold longer in mosquitoes from the populations compared to Rockefeller. Only Pacaraima (2018) remained resistant to $0.12 \%$ deltamethrin. The kdr genotyping revealed the absence of the wild-type Na V S haplotype (1016Val + 1534Phe) in populations from Roraima, meaning that all tested individuals had a genetic background for resistance to pyrethroid. The double kdr Na V R2 haplotype (1016lle + 15434Cys) was under higher frequencies in all populations except Rorainópolis, where this haplotype seems to have arrived recently.

Conclusions These results are important to the knowledge about insecticide resistance status of Ae. aegypti populations from Roraima and will help to improve vector control strategies that may be applied to diverse localities with similar conditions.

\section{Introduction}

The globalization and rapid adaptation of Aedes aegypti to urban conditions are favoring its dispersion around the globe, posing a serious threat to human public health, since this mosquito is vector of arboviruses such as dengue, Zika, chikungunya and urban yellow fever, among others [1]. Roraima is a State in the extreme North of Brazil making international borders with Venezuela and Guyana and is therefore an important Brazilian doorway to the entrance or emergent and re-emergent arbovirus, given the circulation of people between the borders. In addition, population genetic studies suggested that the re-infestation of Ae. aegypti in Brazil likely occurred from Venezuela during the 1970's [2], evidencing the needs of a constant entomological surveillance in Roraima State. 
In 2010 the re-emergence of DENV-4 in Brazil, with a hypothesized origin in Venezuela, was first recorded in Boa Vista, the capital of Roraima, from where rapidly spread to other States in the country: Amazonas and Pará (North), Bahia, Pernambuco and Piauí (Northeast), Rio de Janeiro and São Paulo (Southeast), [3]. Attempting to block the DENV-4 circulation efforts were targeted at the elimination of larval breading sites and the intensification of chemical control, with employment of the insect growth regulator diflubenzuron and the pyrethroid adulticide deltamethrin. This strategy however did not significantly reduce Ae. aegypti infestation and rapidly increased the levels of insecticide resistance to the pyrethroid [4].

Insecticide resistance (IR) is a threat to the control of arboviruses at a global scale [5]. In Brazil, a national wide IR monitoring program coordinated by Ministry of Health has been screening the status of susceptibility to recommended chemicals in Ae. aegypti populations of strategic localities, since 1999 [6]. In previous surveys, resistance to pyrethoid was detected in Ae. aegypti from Boa Vista and Pacaraima [7, 8], probably due to selection of kdr mutations. These mutations are single nucleotide polymorphisms (SNPs) in the voltage gated sodium channel gene $\left(\mathrm{Na}_{\mathrm{V}}\right)$, which encodes a neuronal transmembrane protein that is the target of pyrethroids and DDT. In Ae. aegypti populations from Brazil, there are registers of at least four principal SNPs in the $\mathrm{Na}_{V}$ gene: V410L, $11011 \mathrm{M}, \mathrm{V} 1016 \mathrm{I}$ and $\mathrm{F} 1534 \mathrm{C}[8,9,10]$, whereas the two lasts were more related to resistance to the pyrethroid knockdown effect (kdr) [10]. All Ae. aegypti samples evaluated from Boa Vista and Pacaraima in 2011 harbored kdr mutations, in either $\mathrm{Na}_{\mathrm{V}} \mathrm{R} 1$ $\left(1016 \mathrm{Val}+1534 \mathrm{Cys}^{\mathrm{kdr}}\right)$ or NavR2 (1016llekdr $\left.+1534 \mathrm{Cys}^{\mathrm{kdr}}\right)$ haplotypes [8]. Given resistance to pyrethroids detected in Ae. aegypti populations throughout the country, the MoH replace those compounds to the organophosphate malathion [7], which in Roraima State was introduced in 2015 by governmental campaigns. Pyrethroids however continued probably being applied, especially by household sprays vastly available in market stores, as well as against malaria vectors in endimic regions.

Here we evaluated the profile of resistance of adulticides pyrethroid and organophosphate in Ae. aegypti populations of four important cities of Roraima State, Brazil, in 2016 and 2018, extending the analyses to localities not previously evaluated. The frequency of kdr haplotypes were also investigated.

\section{Methods}

Collections and laboratory rearing conditions. Roraima State has a territory of $224,273.83 \mathrm{Km}^{2}$ and is the less populous Brazilian State, accounting with only $0.29 \%$ of the total population in the country: 605,761 inhabitants, as predicted by the Brazilian Institute of Geography and Statistics [11]. This State is isolated by the Amazon in the south and make international borders with Venezuela and Guyana (Fig. 1). The capital Boa Vista is $741.1 \mathrm{Km}$ distant from Manaus, the capital of Amazonas state, by one only road (BR174). This same road connects Boa Vista to Pacaraima in the border of Venezuela, $2013.5 \mathrm{Km}$ to the North. .

Collections were performed in four of the most important cities of Roraima State, including the capital Boa Vista $\left(02^{\circ} 49^{\prime} 11^{\prime \prime} \mathrm{N}, 60^{\circ} 40^{\prime} 24^{\prime \prime} \mathrm{W}\right)$, Bonfim $\left(02^{\circ} 45^{\prime} 22.25^{\prime \prime} \mathrm{N}, 60^{\circ} 7^{\prime} 6.53^{\prime \prime} \mathrm{O}\right)$ at Northeast in the border with 
Guyana, Pacaraima $\left(04^{\circ} 25^{\prime} 01^{\prime \prime} \mathrm{N}, 61^{\circ} 08^{\prime} 27^{\prime \prime} 0\right)$ at North in the border with Venezuela and Rorainópolis at the South in the border with Amazonas State $\left(00^{\circ} 56^{\prime} 22.62^{\prime \prime} \mathrm{N}, 60^{\circ} 26^{\prime} 21.91^{\prime \prime} \mathrm{O}\right)$. Eggtraps consisted of $800 \mathrm{~mL}$ black plastic cups, containing $300 \mathrm{~mL}$ of a $0.04 \%$ yeast extract solution as an attractant for gravid females, which would lay their eggs in a wood paddle immersed in this solution for 5-7 days. The total of 150 traps were installed in Boa Vista and 50 in each other cities, following standard recommendations [12]. Paddles were shipped to the laboratory at [Núcleo de Pesquisa Observatório da Saúde], Federal University of Roraima State (UFRR), eggs were stimulated to hatch, and larvae reared under laboratory conditions, similarly as described elsewhere [12]. Adults were screened for species identification and maintained in cartoon cages with a $10 \%$ sucrose solution $\mathrm{w} / \mathrm{v}$ offered ad libitum. Anesthetized rats (Rattus norvegicus wistar) were offered to blood feed females in order to produce eggs of an F1 generation, following procedures as recommended in the license \# 12/2015, approved by the ethical committee on animal use of UFRR (CEUA-UFRR). The reference lineage Rockefeller maintained at Laficave/Fiocruz since 1999 [12] was raised in parallel and employed in all assays as a control of insecticide susceptibility and vigor under test conditions.

Entomological surveillance and number of arboviruses cases.

The Brazilian Ministry of Health $(\mathrm{MoH})$ indicates that municipalities are responsible for the entomological surveillance. All municipalities have to provide entomological surveillance data to the $\mathrm{MoH}$, collected by simplified methods of sampling that are rapid and economic, known as LIRAa (the Portuguese anacronym for quick survey of Aedes aegypti indexes), four to six times yearly. The LIRAa is composed by the house $(\mathrm{IH})$, Breteau (IB) and type of recipient (ITR) indexes, which respectively evaluates the percentage of buildings where larvae od Aedes were found in a universe of evaluated buildings, the number of positive breading sites per evaluated buildings, and the number of each type of positive breading sites per the total of all kind of positive breading sites found [13]. The MoF considers that LIRAa IP indexes below $1 \%$ are satisfactory, between 1 and $3.9 \%$ under alert stage and beyond $4 \%$ under risk of dengue outbreak. The register of number of suspected and confirmed cases of arboviruses is of compulsory notification. However, these registers are highly underestimated due to lack or efficacy of health assistance unities in several localities, especially in Roraima State. Here we collected the entomological surveillance IP LIRAa indexes and the number of confirmed cases of arboviruses reported by Gerência de Febre Amarela e Dengue - NCFAD/DVE.CGVS/ Health Secretariat of Roraima State in order to discuss these data in light of our results.

Collections and laboratory rearing conditions.

Bioassays. Bioassays were performed with F1 generation Ae. aegypti females $3-5$ days post-adult emergence with WHO tube tests [14]. Pyrethroid deltamethrin (Sigma Aldrich, St. Louis, USA) technical grade was dissolved in acetone and diluted to the concentrations 0.05 and $0.12 \%$ in silicone oil, as the solvent carrier (Dow Corning, Midland, USA). Malathion (Sangrose Agroquímica, Curitiba, Brazil) emulsion concentrate was diluted in the silicone oil to the concentration $0.7 \%$. Insecticide solutions were applied over a $12 \times 15 \mathrm{~cm}$ filter paper (Whatman Grade 1), as previously described [15]. Mosquitos were exposed 
for 1 hour in the tube chamber with insecticide and then gently transferred to the resting chamber until mortality was register $24 \mathrm{~h}$ later. In addition, knockdown rate was evaluated at each 5 minutes (or 2 minutes for Rockefeller), during the exposition to deltamethrin $0.05 \%$. A probit analysis [16] was employed in order to calculate the time when $50 \%$ of the females were knocked down $\left(\mathrm{KdT}_{50}\right)$. the knockdown resistance ratios ( $R R \mathrm{KdT}_{50}$ ) of the populations were obtained by the quotient between the $\mathrm{KdT}_{50}$ of the populations with Rockefeller.

Kdr genotyping.

DNA was extracted from individual male mosquitoes and genotyped for the single nucleotide polymorphisms (SNPs) in the sites $1016\left(\mathrm{Val}^{+}\right.$or Ile $\left.{ }^{\mathrm{kdr}}\right)$ and $1534\left(\mathrm{Phe}^{+}\right.$or Cys ${ }^{\mathrm{kdr}}$ ) of the voltage gated sodium channel gene $\left(\mathrm{Na}_{\mathrm{V}}\right)$, using exactly the same procedures and reagents as described in [17]. As these sites are linked in the same gene, both SNPs were considered in order to define the genotype frequencies for a single locus [8]. The haplotypes usually found in Ae. aegypti populations from Brazil are the wild-type $\mathrm{Na}_{\mathrm{V}} \mathrm{S}$ (1016Val + 1534Phe) and the kdrs Na $\mathrm{V} 1$ (1016Val + 1534Cys) and Nav R2 (1016lle + 1534Cys), whereas the possible genotypes are SS, SR1, SR2, R1R1, R1R2 and R2R2. As kdr mutations act as a recessive trait, individuals resistant to pyrethroid based on this target site mechanism present one of the genotypes R1R1, R1R2 or R2R2. The NavR2 haplotype confers higher levels of resistance [15].

\section{Results}

Entomological surveillance and number of arboviruses cases in Roraima. Figure 2 shows the Ae. aegypti infestation index registered between 2015 and 2018 in the four localities from Roraima State here studied. At least one out of the four yearly surveys indicated that all these municipalities were under risk of dengue outbreak ( $\mathrm{PI}>4 \%$ ) every year. The highest infestation index was observed in Bonfim, during the third survey of 2017 , however Rorainópolis presented the highest yearly averages. More than $30 \%$ of larval breeding sites observed by health agents in Roraima have been those classified as "D2": garbage (disposable plastic containers, bottles, cans, iron scraps), followed by "B": non-fixed deposits (plant vases, plates, flasks, drink fountains) in around $25 \%$ amongst all breeding sites.

During this same period, the highest number of dengue in Roraima State was registered in 2015, while chikungunya and Zika were mostly recorded in 2017 (Figure 3). The number of arboviruses cases were by large higher in the capital Boa Vista. The continuous registers of arboviruses cases are justified by the high infestation indexes of Ae. aegypti (Figure 2). The number of confirmed arboviruses cases and incidence/ 100,000 inhabitants between 2015 and 2018 is displayed in Additional File S1. 
Bioassays. We evaluated the susceptibility status of Ae. aegypti from four Roraima municipalities to the adulticides pyrethroid deltamethrin $(0.05$ and $0.12 \%)$ and to the organophosphate malathion $(0.7 \%)$, in 2016 and 2018. It is of note that only Ae. aegypti resulted from these collections, regardless to a recent register of Ae. albopictus at a rural area in Rorainopolis. In 2016, except Rorainópolis, which achieved $100 \%$ mortality, populations presented some survival to malathion $0.7 \%$, however in all cases above $90 \%$ (Figure 3). There was a reduction in the mortality levels in 2018 in all localities, ranging from $84.8 \%$ in Pacaraima to $94.2 \%$ in Rorainópolis (Table 1). Therefore, Pacaraima and Boa Vista were classified as resistant to malathion in 2018.

In regard to the pyrethroid, all populations were considered resistant both in 2016 and 2018 when evaluated with $0.05 \%$ deltamethrin. From 2016 to 2018 there was an increase in the mortality in Boa Vista (64.0 to $86.6 \%$ ) and Bonfim (31.2 to 78.5\%), and a decrease in Rorainópolis (89.9 to $74.3 \%$ ) and Pacaraima (78.3 to $61.7 \%$ ). A higher dosage of deltamethrin (0.12\%) was tested in parallel. As expected, this higher concentration increased the mortality in all cases, however did not cause $100 \%$ of mortality in any population (Figure 4-A). We also evaluated the rate of knockdown during the time of exposition to deltamethrin $0.05 \%$ for $1 \mathrm{~h}$ (Additional file Figure S2). According to a probit analysis, while $50 \%$ of the Rockefeller females were knocked down ( $\left.K d T_{50}\right)$ in 11.9 minutes, the same took between 29.6 (Boa Vista, 2018) and 69.9 (Bonfim, 2016) minutes in the populations (Table 2). Nevertheless, the $K d T_{50}$ values of all populations were similar if we consider the IC95\% interval, except Bonfim in 2016 which presented a higher $K d \mathrm{~T}_{50}$ (Figure 4-B). The knockdown-resistance ratio $\left(K d T-\mathrm{RR}_{50}\right)$ of the populations ranged from 2.5 and 5.9 (Table 2).

Kdrgenotyping. We successfully genotyped 275 Ae. aegypti individuals from Roraima State for both 1016 (Val+ or lle $\mathrm{e}^{k d \eta}$ ) and 1534 (Phe ${ }^{+}$or Cys ${ }^{k d \eta}$ ) sites. All tested individuals showed a kdr mutation in at least one site, presenting the genotypes homozygotes for the $1534 \mathrm{kdr}$ (R1R1) and the double $k d r$ $1016+1534$ (R2R2), in addition to the heterozygotes (R1R2). Therefore there was no evidence of occurrence of the wild-type haplotype S (1016 Val $+1534 \mathrm{Phe})$ in Ae. aegypti populations from Roraima (Figure 5).

The double $k d r$ haplotype R2 (1016 lle + 1534 Cys) was in higher frequency than R1 (1016 Val + 1534 Cys) in Boa Vista (2018), Bonfim (2016 and 2018) and Pacaraima (2016 and 2018) (Table 3). In Rorainópolis R2 was under low frequencies (8 and 11\%, respectively in 2016 and 2018), only appearing in heterozygotes R1R2. In all cases the genotipic frequencies did not deviated from the HardyWeinberg Equilibrium assumption (Table 3). 
When comparing the genotypic frequencies between 2016 and 2018 collections, the genotype R2R2 decreased in Boa Vista (25 to 18.6\%), Bonfim (45 to 36.7\%) and specially in Pacaraima (45.5 to $20.7 \%$ ). Indeed, as aforementioned the levels of morality to deltamethrin $0.05 \%$ increased in Boa Vista and Bonfim, however decreased in Pacaraima (Figure 4-A).

We also genotyped some individuals dead (susceptible) and alive (resistant) $24 \mathrm{~h}$ after $1 \mathrm{~h}$ of exposition to deltamethrin $0.05 \%$ from Boa Vista and Bonfim. Although the number of samples was small (41 alive and 14 dead), in the resistant group the R1R1 genotype was absent, while R2R2 was more represented in this group (Figure $5-\mathrm{C}$ ). This corroborates the hypothesis that the $\mathrm{Na}_{V} \mathrm{R} 2$ allele potentially confers higher levels of resistance to pyrethroid.

\section{Discussion}

Here we showed that Ae. aegypti populations from Roraima State, Brazil collected in 2016 and 2018, were resistant to the pyrethroid deltamethrin and is the process of becoming resistant to the organophosphate malathion.

The larvicide organophosphate temephos was distributed from the MoH to Roraima State between 2004 and 2013 [7] and the IR monitoring network detected resistance to temephos in Ae. aegypti from Boa Vista in 2002, 2003, 2004 and 2007 [5, 18], but not in 2010 [4]. Mosquitoes from Pacaraima were resistant to temephos in 2011 [19]. The disseminated resistance to tempephos in the whole country made the $\mathrm{MoH}$ substitute temephos for the biolarvicide Bacillus thuringiensis var. israelensis (Bt) and later for IGR insecticides, such as the benzophenyl urea inhibitors of chitin synthesis diflubenzuron and novaluron, which started being applied in Roraima in 2010 [7].

Resistance to pyrethroid in Ae. aegypti from Roraima State was already high in previous evaluations. The first registers were in 2007 and 2010, when the capital Boa Vista was detected as resistant to pyrethroid $[4,18]$. In 2011 Pacaraima presented the second most high resistance ration $\left(R R_{95}=60.3\right)$ detected that year in the whole country [7]. Here we showed that even two years after pyrethroid governmental application was substituted by malathion, resistance to pyrethroid is maintained in the four localities evaluated.

The Brazilian $\mathrm{MoH}$ started replacing pyrethroids for the organophosphate malathion in ultra-low volumebased applications against Ae. aegypti in 2010 [7], although pyrethroids are still used in campaigns against other vectors, such as anophelines, phlebotomines and tritomines. In Roraima State the first 
stock of malathion was received in December 2015. Here we showed that all the four populations collected in 2016 , although not $100 \%$ killed to malathion $0.7 \%$, had mortality above $90 \%$, then not classified as resistant. The rate of mortality decreased by under $90 \%$ in Bonfim and Pacaraima two years later and were therefore classified as resistant to malathion. Differently for what occurs with pyrethroids, which are the active ingredient of household sprays and other governmental campaigns, the probable only source of organophosphate pressure was the governmental campaigns with malathion at that moment. The larvicide temephos is in theory not applied in Roraima since 2013, when was definitely substituted by IGRs [7]. The most recent data about temephos resistance in Roraima indicated resistance ratios $\left(\mathrm{RR}_{50}\right)$ of 2.0 in Boa Vista (2010) and 4.3 in Pacaraima (2011), which were not considered high levels of resistance $[4,18]$. Anyway, we cannot reject the possibility that mechanisms prior selected by temephos and pyrethroids are conferring cross resistance to malathion, as reported in some classical studies. For instance, a laboratory strain of Culex quinquefasciatus selected for temephos resistance in larvae developed cross resistance to several organophosphate adulticides, including malathion [20]. On the other hand, in Guadeloupe and Saint Martin Caribbean islands, Ae. aegypti populations developed high levels of resistance to temephos (8.9 to 33.1 -fold) but low levels to malathion (1.7 to 4.4 -fold) [21]

In regard to the possible mechanisms selected for insecticide resistance, alteration in the activity of GST and Esterase enzymes were detected in Boa Vista (2007) and Pacaraima (2011). Reduced activity of acetylcholinesterase enzyme was also observed in Pacaraima (2011) [7]. Lines of Ae. aegypti Brazilian populations that acquired resistance to malathion though selection pressure in the laboratory exhibited increased activity of GST, MFO P450 and esteresas, as determined by bichoemical analyses [22]. Over expression of genes related to metabolic resistance were detected in Ae. aegypti populations from Caribe, such as French Guyana and French West Indies islands [21,23]. These metabolic related mechanisms however are difficult to associate with a specific class of insecticide. On the other hand, those high levels of resistance to pyrethroids in Roraima could be partially justified by the absence of the wild-type $\mathrm{Na}_{\mathrm{V}} \mathrm{S}$ haplotype, already noted 2010 and 2011 in Boa Vista and Pacaraima [8]. The wild-type $\mathrm{Na}_{\mathrm{V}} \mathrm{S}$ haplotype is still missing, with predominance of the double $k d r \mathrm{Na}_{\mathrm{V}} \mathrm{R} 2$ (1016lle + 1534Cys), except in Rorainópolis, where the $\mathrm{Na}_{\mathrm{V}} \mathrm{R} 1$ (1016Val $\left.+1534 \mathrm{Cys}\right)$ predominates. We corroborated that $\mathrm{Na}_{\mathrm{V}} \mathrm{R} 2$ confreres higher levels of resistance to pyrethroid [15], once homozygotes R2R2 individuals were exclusively among survivals in bioassays with Boa Vista and Bonfim. The remaining high levels of resistance to pyrethroid even after the change for malathion in Roraima can be associated with the high prevalence of domestic use of insecticides, all composed of pyrethroids and easily acquired in local markets, as reported in other Brazilian states $[17,24]$. In addition, we cannot neglect the migration of Ae. aegypti resistant populations from the neighbor countries and measures adopted against other vector borne diseases. In the border between Pacaraima (Brazil) and Santa Helena (Venezuela) there is an intense control of malaria, where pyrethroids are employed against Anopheles even in the urban area, thus submitting also Ae. aegypti to that selection pressure. In Bonfim it is also usual to find pyrethroid sprays acquired in Lethen City, in the Guyanese side of the border. 
We speculate that the $k d r \mathrm{Na}_{\mathrm{V}} \mathrm{R} 2$ haplotype in Ae. aegypti populations in that region should have migrated mostly from Venezuela, once it has been registered in Roraima State at least since 2010, when was absent or under low frequencies in the neighbors Amazonas and Pará States [8]. Interestingly, Rorainópolis is the only municipality in Roraima with occurrence of Ae. albopictus, however registered in areas in the border with Amazonas State, where that species has been registered since 2015 (CGVSSESAU-RR). Therefore, it seems that the ecological transition zone between the biomes Amazon Forest to the south and the savannah-like Lavrado to the north has been limiting the dispersion of Ae. aegypti towards Amazonas State, whilst Ae. albopictus is avoided to invade the capital Boa Vista.

Besides new promising tools of Ae. aegypti control, such as Wolbachia, RIDL and pyriproxyfen autodissemination stations [25] currently in advance tested in Brazil [26, 27, 28], insecticides should play an important role in a long term in order to rapid decrease the densities of a target population and consequently mitigate the cycle of the arboviruses. Therefore it is of prime importance that a constant monitoring of insecticide resistance be inherent of the chemical control strategy in order to guide authorities about which products should have the best efficacy.

\section{Abbreviations}

DDT: dichlorodiphenyltrichloroethane; $\mathrm{Na}_{v}$ : voltage-gated sodium channel gene; WHO: World Health Organization; MoH: Ministry of Health; LIRAa: quick survey of Aedes aegypti indexes; IGR: insect growth regulator; $K d r$. knockdown resistance; RR: resistance ratio; $K d T$ : time of knockdown; SNP: single nucleotide polymorphism.

\section{Declarations}

\section{Acknowledgements}

We thank Pedro Eduardo Lima Siqueira and Emilly Habert (UFRR) for their support with the bioassays and Heloisa Diniz, Instituto Oswaldo Cruz, for taking care of the figures in this publication. 


\section{Declarations}

\section{Ethics approval and consent to participate}

Animals (Rattus norvegicus wistar) were used in this study in order to blood feed female mosquitos to produce laboratory colonies. Animals were maintained and anesthetized prior to blood feeding, accordingly to the procedures indicated by the ethical committee on animal use of Federal University of Roraima (CEUA-UFRR), approved license \# 12/2015.

\section{Consent for publication}

Not applicable.

\section{Availability of data and materials}

All data generated or analyzed during this study are included in this published article [and its supplementary information files]. Any additional dataset are available upon request to the corresponding author.

\section{Competing interests}

The authors declare that they have no competing interests.

\section{Funding}

National Institute of Health (NIH, Grant no. U01 Al115595), Instituto Nacional de Ciência e Tecnologia em Entomologia Molecular (INCT-EM, Grant no. 465678/2014-9), and Fundação de Amparo à Pesquisa do Estado do Rio de Janeiro (FAPERJ, Grant no. E-26/202.795/2019).

\section{Author contributions:}

Conceptualization: RLNH, AJM 
Data curation: RLNH, LC

Formal Analysis: RLNH, AJM

Funding acquisition: RLNH, JBPL, AJM

Investigation: RLNH, AJM, JML, NCVA

Methodology: LC, JBPL, AJM

Project administration: AJM

Resources: JBPL, AJM

Supervision: AJM

Validation: AJM

Visualization: all authors

Writing - original draft: RLNH, AJM

Writing - review \& editing: all authors

\section{All authors read and approved the final manuscript.}

\section{References}

1. Kraemer MUG, Reiner RC, Jr., Brady OJ, Messina JP, Gilbert M, Pigott DM, et al. Past and future spread of the arbovirus vectors Aedes aegypti and Aedes albopictus. Nat Microbiol. 2019;4 5:854-63; doi: 10.1038/s41564-019-0376-y. https://www.ncbi.nlm.nih.gov/pubmed/30833735.

2. Kotsakiozi P, Gloria-Soria A, Caccone A, Evans B, Schama R, Martins AJ, et al. Tracking the return of Aedes aegypti to Brazil, the major vector of the dengue, chikungunya and Zika viruses. PLoS Negl Trop Dis. 2017;11 7:e0005653; doi: 10.1371/journal.pntd.0005653. https://www.ncbi.nlm.nih.gov/pubmed/28742801. 
3. de Souza RP, Rocco IM, Maeda AY, Spenassatto C, Bisordi I, Suzuki A, et al. Dengue virus type 4 phylogenetics in Brazil 2011: looking beyond the veil. PLoS Negl Trop Dis. 2011;5 12:e1439; doi: 10.1371/journal.pntd.0001439. http://www.ncbi.nlm.nih.gov/pubmed/22216365.

4. Maciel-de-Freitas R, Avendanho FC, Santos R, Sylvestre G, Araujo SC, Lima JB, et al. Undesirable consequences of insecticide resistance following Aedes aegypti control activities due to a dengue outbreak. PloS one. 2014;9 3:e92424; doi: 10.1371/journal.pone.0092424. http://www.ncbi.nlm.nih.gov/pubmed/24676277.

5. Moyes CL, Vontas J, Martins AJ, Ng LC, Koou SY, Dusfour I, et al. Contemporary status of insecticide resistance in the major Aedes vectors of arboviruses infecting humans. PLoS Negl Trop Dis. 2017;11 7:e0005625; doi: 10.1371/journal.pntd.0005625. https://www.ncbi.nlm.nih.gov/pubmed/28727779.

6. Braga IA, Valle D. Aedes aegypti: vigilância, monitoramento da resistência e alternativas de controle no Brasil. Epidemiol Serv Saúde. 2007;16:295-302.

7. Valle D, Bellinato DF, Viana-Medeiros PF, Lima JBP, Martins Junior AJ. Resistance to temephos and deltamethrin in Aedes aegypti from Brazil between 1985 and 2017. Mem Inst Oswaldo Cruz. 2019;114:e180544; doi: 10.1590/0074-02760180544. https://www.ncbi.nlm.nih.gov/pubmed/31038548.

8. Linss JG, Brito LP, Garcia GA, Araki AS, Bruno RV, Lima JB, et al. Distribution and dissemination of the Val1016lle and Phe1534Cys Kdr mutations in Aedes aegypti Brazilian natural populations. Parasit Vectors. 2014;7 1:25; doi: 10.1186/1756-3305-7-25. http://www.ncbi.nlm.nih.gov/pubmed/24428880.

9. Martins AJ, Brito LP, Linss JG, Rivas GB, Machado R, Bruno RV, et al. Evidence for gene duplication in the voltage-gated sodium channel gene of Aedes aegypti. Evolution, medicine, and public health. 2013;2013 1:148-60; doi: 10.1093/emph/eot012. http://www.ncbi.nlm.nih.gov/pubmed/24481195.

10. Haddi K, Tome HVV, Du Y, Valbon WR, Nomura Y, Martins GF, et al. Detection of a new pyrethroid resistance mutation (V410L) in the sodium channel of Aedes aegypti: a potential challenge for mosquito control. Scientific reports. 2017;7:46549; doi: 10.1038/srep46549.

https://www.ncbi.nlm.nih.gov/pubmed/28422157.

11. IBGE: IBGE Cidades. https://cidades.ibge.gov.br/brasil/rr/panorama (2019). 2019.

12. Lima JB, Da-Cunha MP, Da Silva RC, Galardo AK, Soares Sda S, Braga IA, et al. Resistance of Aedes aegypti to organophosphates in several municipalities in the State of Rio de Janeiro and Espirito Santo, Brazil. Am J Trop Med Hyg. 2003;68 3:329-33. http://www.ncbi.nlm.nih.gov/pubmed/12685640.

13. Brasil: [Levantamento rápido de índices para Aedes aegypti - LIRAa - Para vigilância entomológica do Aedes aegypti no Brasil: metodologia para avaliação dos índices de Breteau e Predial e tipo de recipientes] Edited by Ministério da Saúde SdVneSd, Departamento de Vigilância das Doenças Transmissíveis. Brasília: Ministério da Saúde; 2013.

14. WHO. Monitoring and managing insecticide resistance in Aedes mosquito populations - Interim guidance for entomologists. vol. WHO/ZIKV/VC/16.1. Geneva: World Health Organization; 2016. 
15. Brito LP, Carrara L, Freitas RMd, Lima J, \#xe9, Pereira B, et al. Levels of Resistance to Pyrethroid among Distinct kdr Alleles in Aedes aegypti Laboratory Lines and Frequency of kdr Alleles in 27 Natural Populations from Rio de Janeiro, Brazil. BioMed research international. 2018;2018:10; doi: 10.1155/2018/2410819. https://doi.org/10.1155/2018/2410819.

16. Raymond M. Presentation d'une programme d'analyse logprobit pour microordinateur cahiers Orstrom. Sér Ent Med Parasitol. 1985;22:117-21.

17. Macoris ML, Martins AJ, Andrighetti MTM, Lima JBP, Valle D. Pyrethroid resistance persists after ten years without usage against Aedes aegypti in governmental campaigns: Lessons from Sao Paulo State, Brazil. PLoS Negl Trop Dis. 2018;12 3:e0006390; doi: 10.1371/journal.pntd.0006390. https://www.ncbi.nlm.nih.gov/pubmed/29601580.

18. Belinato TA, Martins A, Valle D. Fitness evaluation of two Brazilian Aedes aegyptifield populations with distinct levels of resistance to the organophosphate temephos. Mem I Oswaldo Cruz. 2012;in press.

19. Bellinato DF, Viana-Medeiros PF, Araujo SC, Martins AJ, Lima JB, Valle D. Resistance Status to the Insecticides Temephos, Deltamethrin, and Diflubenzuron in Brazilian Aedes aegypti Populations. BioMed research international. 2016;2016:8603263; doi: 10.1155/2016/8603263. http://www.ncbi.nlm.nih.gov/pubmed/27419140.

20. Peiris HTR, Hemingway J. Temephos resistance and the associated cross-resistance spectrum in a strain of Culex quinquefasciatus Say (Diptera: Culicidae) from Peliyagoda, Sri Lanka. B Entomol Res. 1990;80 1:49-55; doi: https://doi.org/10.1017/S0007485300045910.

21. Goindin D, Delannay C, Gelasse A, Ramdini C, Gaude T, Faucon F, et al. Levels of insecticide resistance to deltamethrin, malathion, and temephos, and associated mechanisms in Aedes aegypti mosquitoes from the Guadeloupe and Saint Martin islands (French West Indies). Infect Dis Poverty. 2017;6 1:38; doi: 10.1186/s40249-017-0254-x. https://www.ncbi.nIm.nih.gov/pubmed/28187780.

22. Viana-Medeiros PF, Bellinato DF, Valle D. Laboratory selection of Aedes aegypti field populations with the organophosphate malathion: Negative impacts on resistance to deltamethrin and to the organophosphate temephos. PLoS Negl Trop Dis. 2018;12 8:e0006734; doi:

10.1371/journal.pntd.0006734. https://www.ncbi.nlm.nih.gov/pubmed/30125295.

23. Dusfour I, Zorrilla P, Guidez A, Issaly J, Girod R, Guillaumot L, et al. Deltamethrin Resistance Mechanisms in Aedes aegypti Populations from Three French Overseas Territories Worldwide. PLoS Negl Trop Dis. 2015;9 11:e0004226; doi: 10.1371/journal.pntd.0004226. http://www.ncbi.nlm.nih.gov/pubmed/26588076.

24. Garcia GA, Sylvestre G, Aguiar R, Costa GB, Martins AJ, Lima JBP. Matching the genetics of released and local Aedes aegypti populations is critical to assure Wolbachia invasion. PLoS Negl Trop Dis. 2019;131; doi: 10.1371/journal.pntd.0007023. https://doi.org/10.1371/journal.pntd.0007023.

25. Achee NL, Grieco JP, Vatandoost H, Seixas G, Pinto J, Ching-Ng L. Alternative strategies for mosquitoborne arbovirus control. PLoS Negl Trop Dis. 2019;131; doi: 10.1371/journal.pntd.0006822. https://doi.org/10.1371/journal.pntd.0006822. 
26. O'Neill SL, Ryan PA, Turley AP, Wilson G, Retzki K, Iturbe-Ormaetxe I, et al. Scaled deployment of Wolbachia to protect the community from dengue and other Aedes transmitted arboviruses [version 3; peer review: 2 approved]. Gates Open Res. 2019;2:36; doi: 10.12688/gatesopenres.12844.3. https://www.ncbi.nlm.nih.gov/pubmed/30596205.

27. Evans BR, Kotsakiozi P, Costa-da-Silva AL, loshino RS, Garziera L, Pedrosa MC, et al. Transgenic Aedes aegypti Mosquitoes Transfer Genes into a Natural Population. Scientific reports. 2019;9 1:13047; doi: 10.1038/s41598-019-49660-6. https://www.ncbi.nlm.nih.gov/pubmed/31506595.

28. Abad-Franch F, Zamora-Perea E, Ferraz G, Padilla-Torres SD, Luz SL. Mosquito-disseminated pyriproxyfen yields high breeding-site coverage and boosts juvenile mosquito mortality at the neighborhood scale. PLoS Negl Trop Dis. 2015;9 4:e0003702; doi: 10.1371/journal.pntd.0003702. https://www.ncbi.nlm.nih.gov/pubmed/25849040.

\section{Tables}

Due to technical limitations, tables are only available as a download in the supplemental files section

\section{Figures}
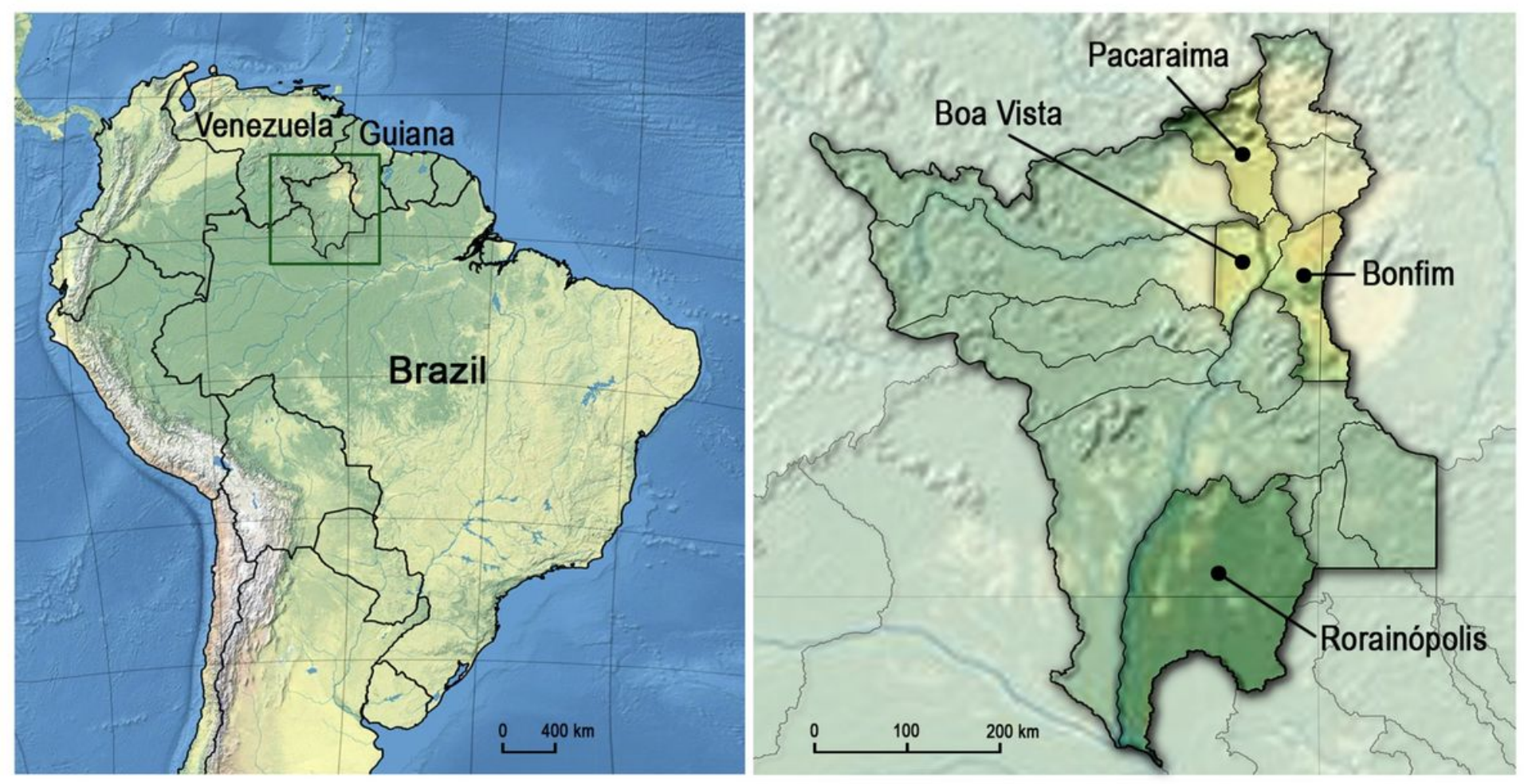

Figure 1 

Guyana. Eggs of Aedes were collected in Boa Vista, Bonfım, Pacaraima and Rorainópolis, indicated in the magnified square.

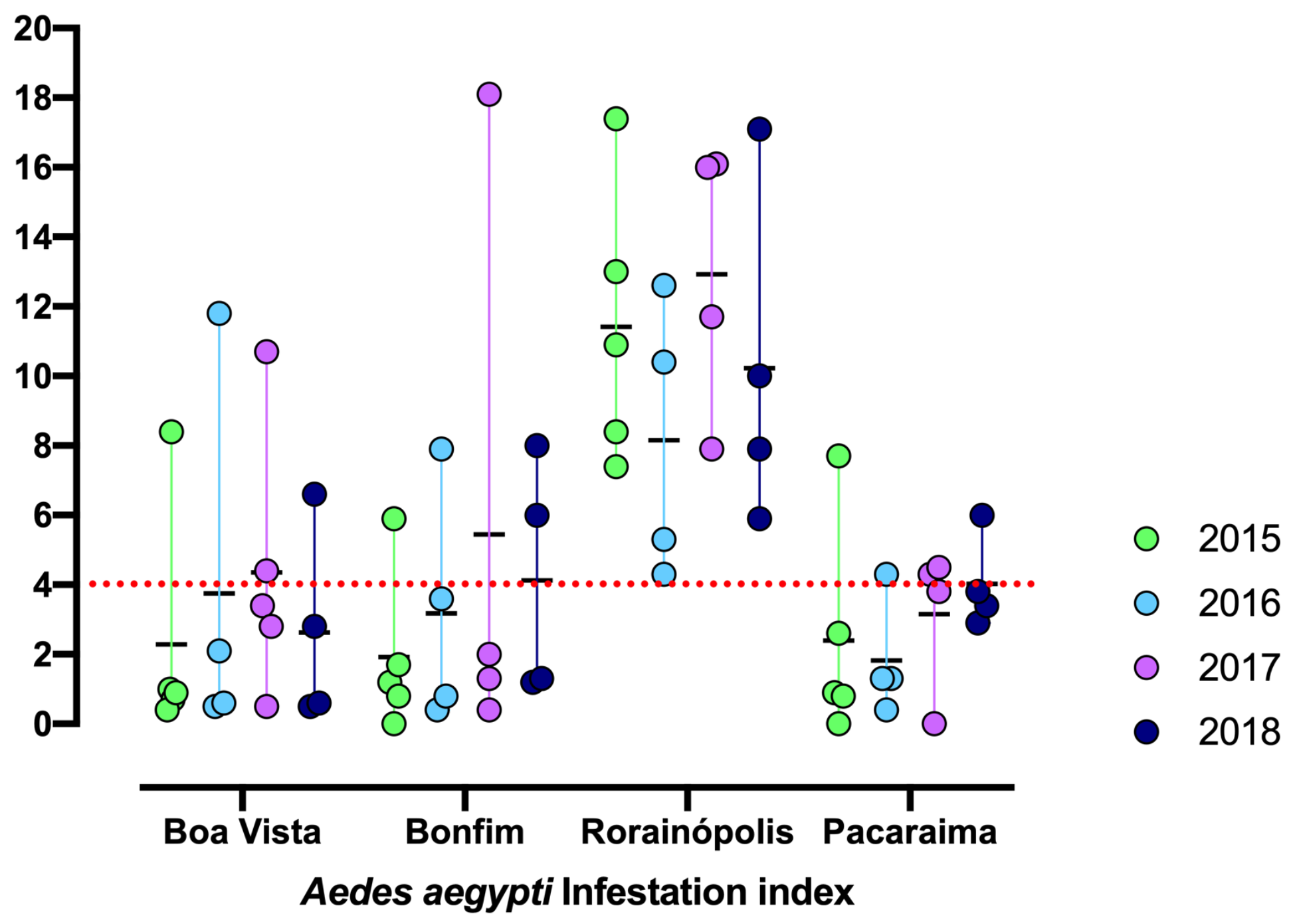

Figure 2

Infestation index of Aedes aegypti in Roraima State during 2015-2018. Each dot represents the House Index $(\mathrm{HI})$, which is the percentage of visited houses where some larva of Aedes was found, during one quick survey of Aedes aegypti indexes (LIRAa). Lines connect the minimum and maximum yearly $\mathrm{HI}$ values, with a horizontal bar representing the year average. An infestation index above $4 \%$ indicates risk of dengue outbreak (dotted red line). Only the localities evaluated in this study were represented.

Coordenação Geral de Vigilância Sanitária/ Departamento de Vigilância Epidemiológica/ Secretaria de Estado de Saúde de Roraima. 

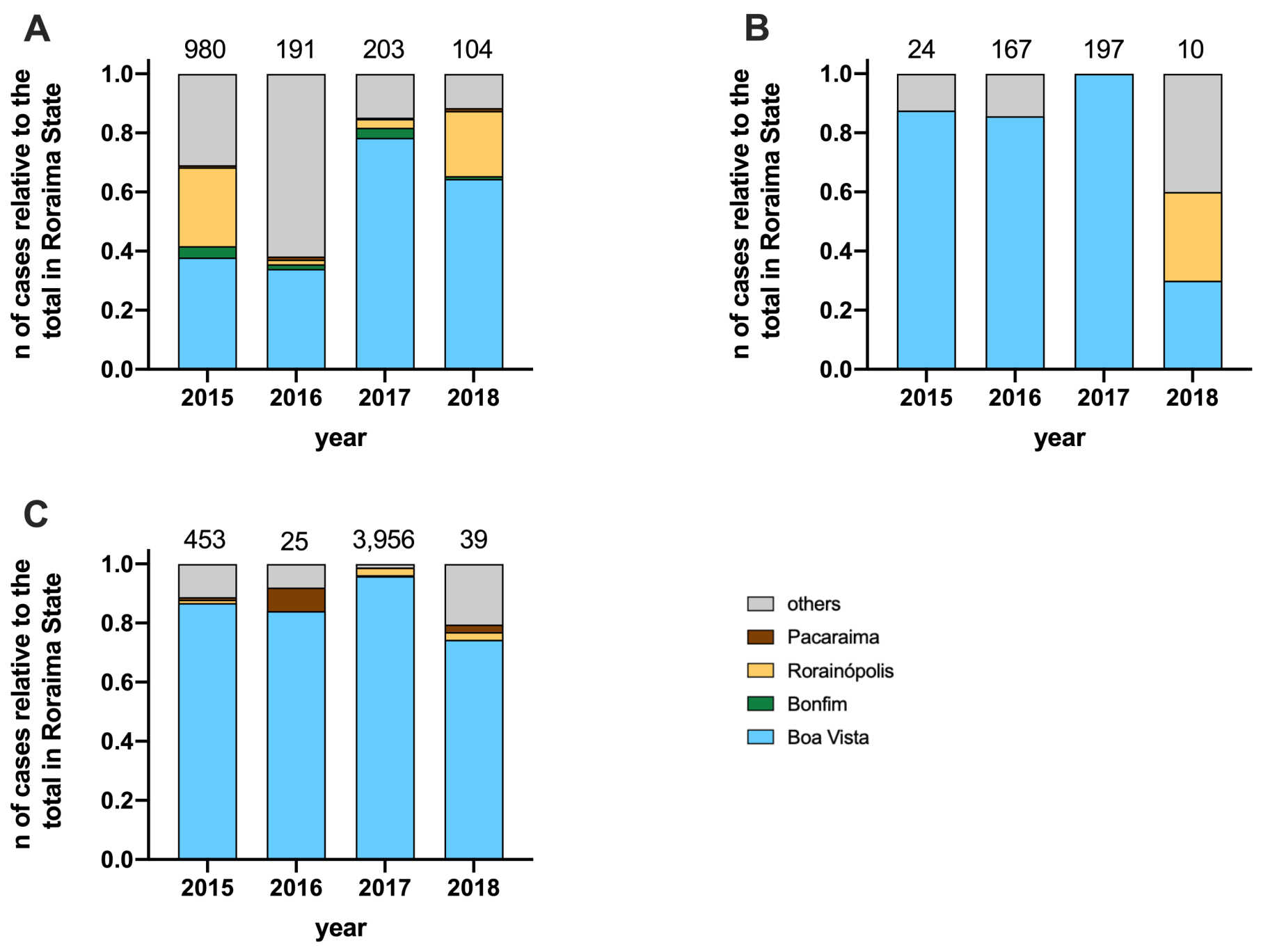

Figure 3

Cases of arboviruses registered in Roraima State during 2015-2018. Data of dengue, Zika and chikungunya in each locality were represented in proportional to the total number of cases yearly registered in the State (numbers above the bars). Source: Coordenação Geral de Vigilância Sanitária/ Departamento de Vigilância Epidemiológica/ Secretaria de Estado de Saúde de Roraima. 


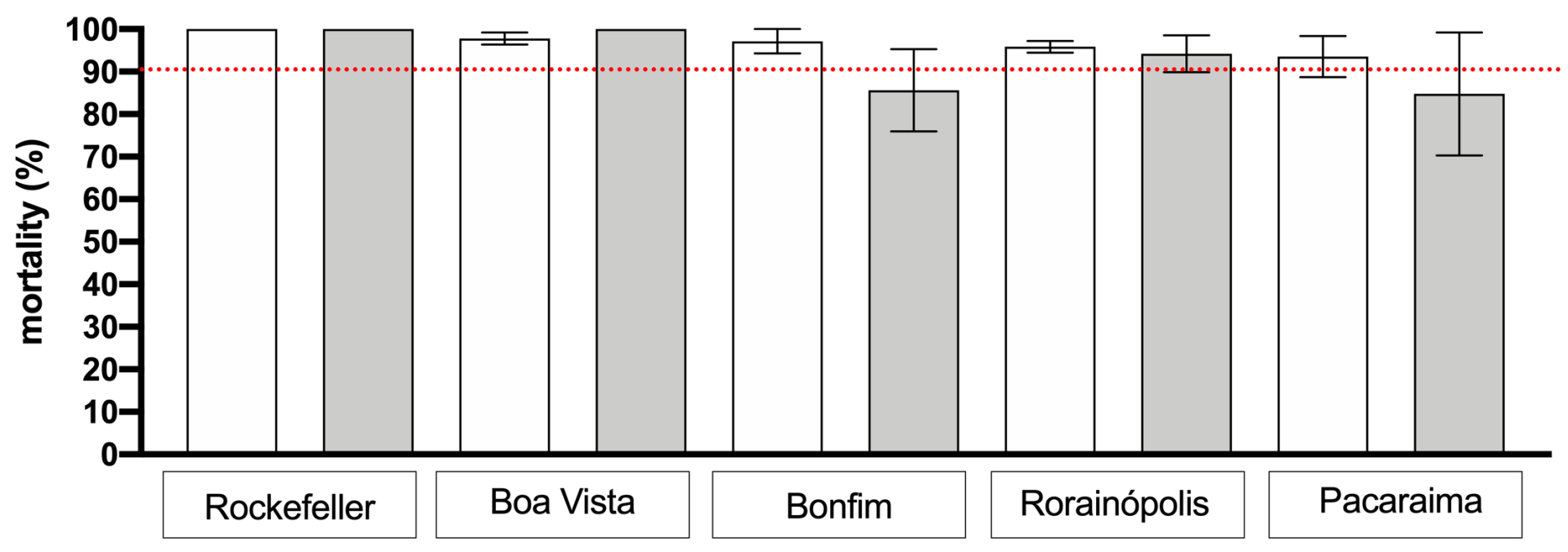

Legend $\square 2016 \quad \square 2018$

Figure 4

Evaluation of Ae. aegypti from Roraima to the organophosphate malathion. Bars indicate the mean percentage of mortality (with standard error of mean), $24 \mathrm{~h}$ post $1 \mathrm{~h}$ of exposition to malathion $0.7 \%$, for Ae. aegypti populations from Boa Vista, Bonfım, Rorainópolis and Pacaraima, additional to the reference lineage Rockefeller. Assays were performed for the collections in 2016 and 2018. Populations with mortality beyond $90 \%$ (red dotted line) are classified as resistant to the insecticide. 


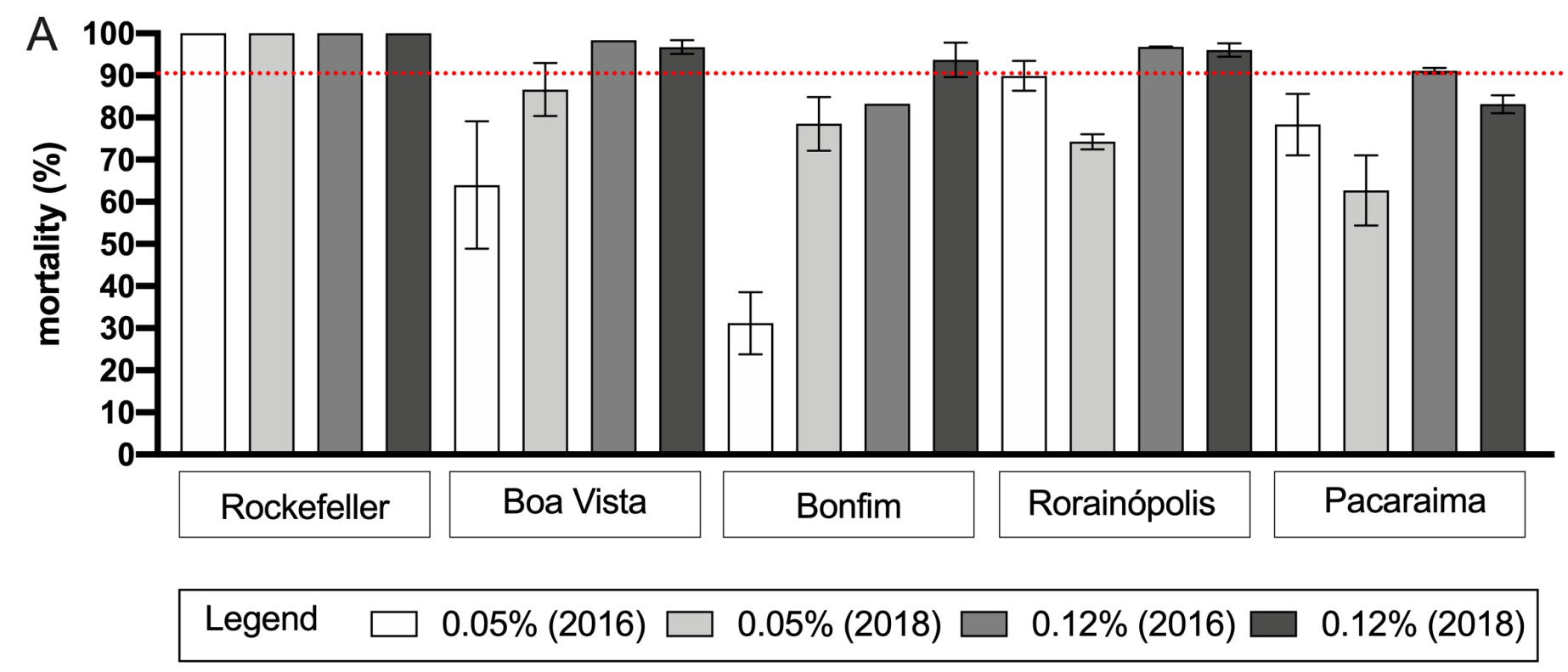

B

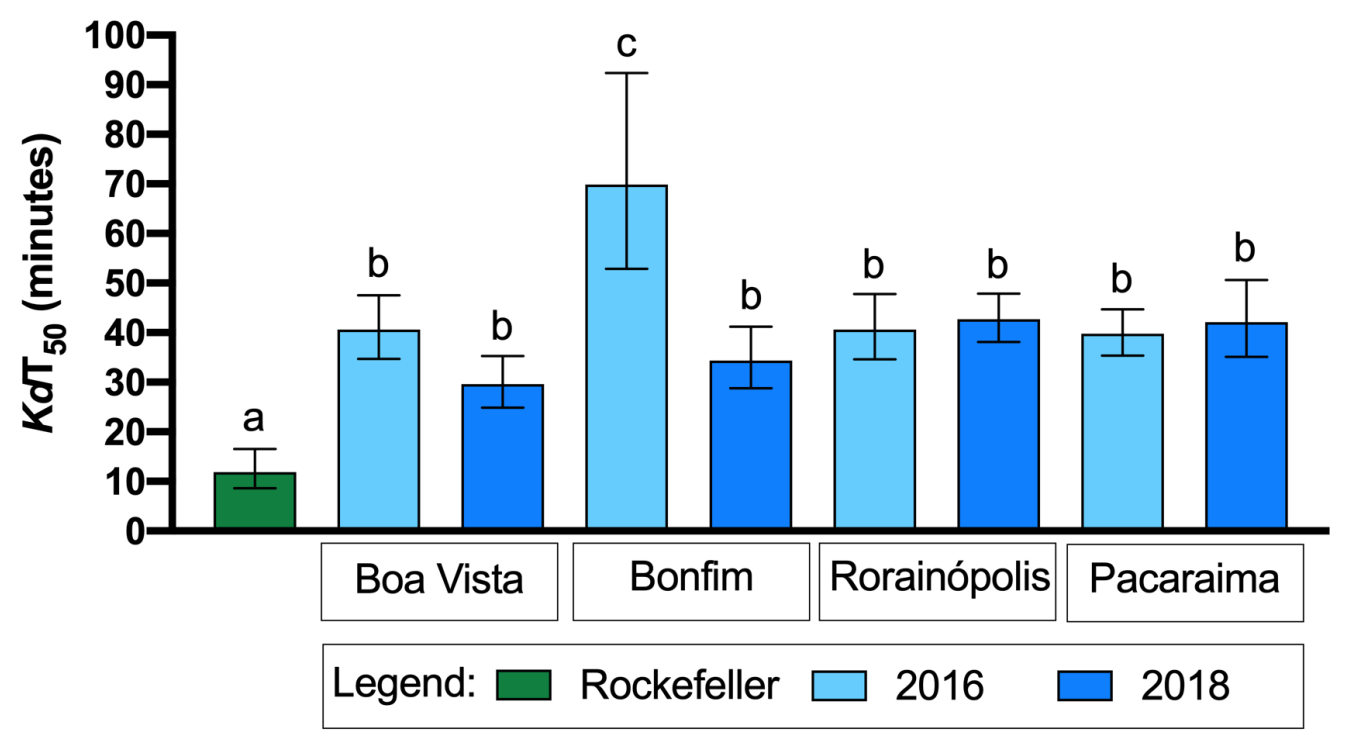

\section{Figure 5}

Evaluation of Ae. aegypti from Roraima to the pyrethroid deltamethrin. A-Bars indicate the mean percentage of mortality (with standard error of mean), 24h post $1 \mathrm{~h}$ of exposition to deltamethrin 0.05 and $0.12 \%$, for Ae. aegypti populations from Boa Vista, Bonfim, Rorainópolis and Pacaraima, additional to the reference lineage Rockefeller. Assays were performed for the collections in 2016 and 2018. Populations with mortality beyond $90 \%$ (red dotted line) are classified as resistant to the insecticide. B - Median time, in minutes, that $50 \%$ of the females exposed to deltamethrin $0.05 \%$ toke to knockdown (KdT50), with $95 \%$ confidence interval (IC95). Similar letters indicate similar times (overlapped IC95 intervals). 

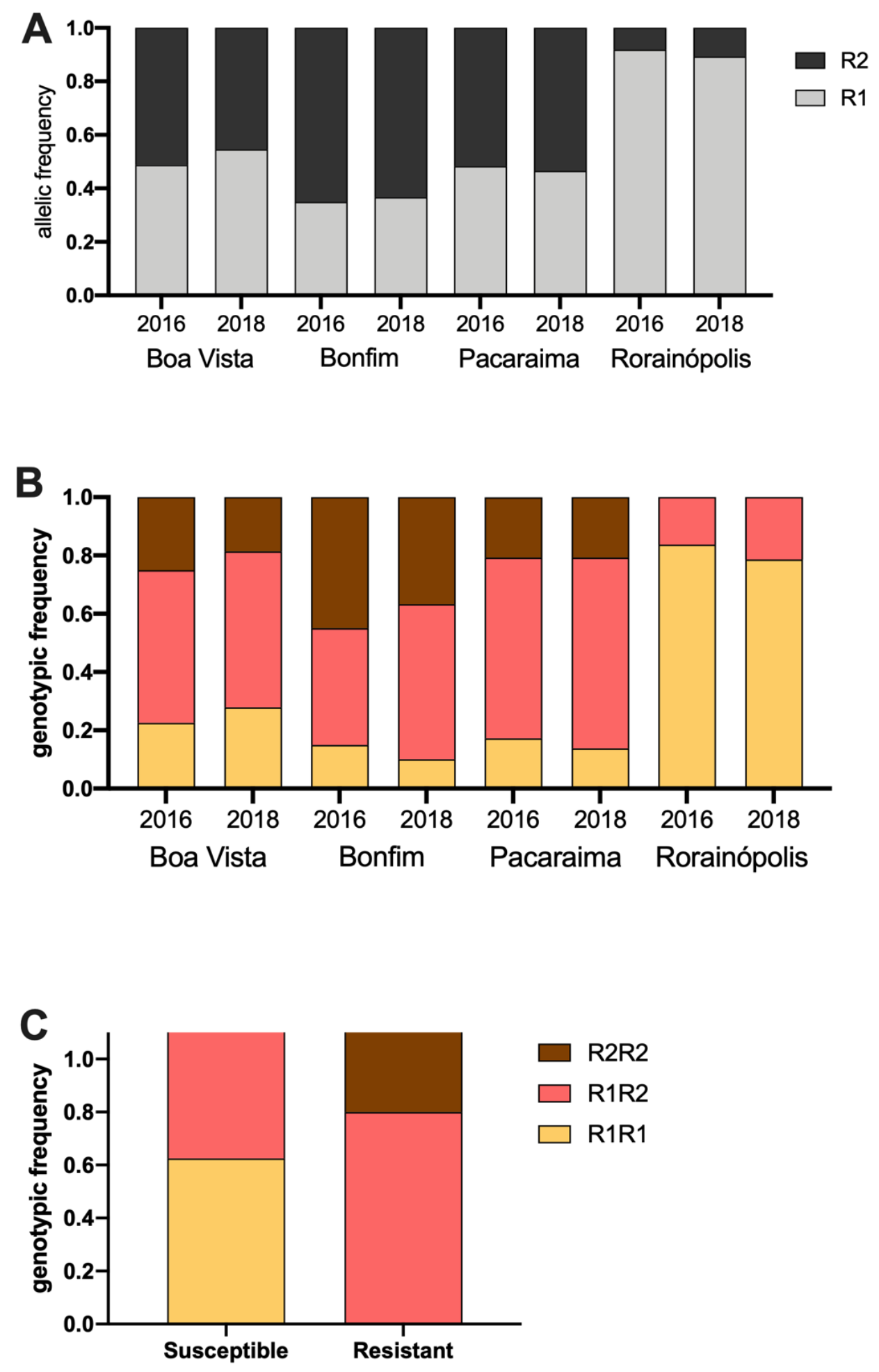

Figure 6

Genotyping of kdr mutations in Ae. aegypti populations from Roraima. A-Allelic frequencies of the kdr haplotypes NaVR1 (1016Val + 1534Cyskdr) and NaVR2 (1016llekdr + 1534Cyskdr) in Ae. aegypti populations from Boa Vista, Bonfim, Rorainópolis and Pacaraima. There was no evidence of presence ot the wilt-type haplotype NaVS (1016Val + 1534Phe) in both 2016 and 2018 sampling years. B - Genotypic 
frequencies of each population in both years. C - Genotypic frequencies of a sampling of Ae. aegypti from Boa Vista and Bonfim (2016) that died (Susceptible) and survived (Resistant) to deltamethrin $0.05 \%$.

\section{Supplementary Files}

This is a list of supplementary files associated with this preprint. Click to download.

- Graphicabstract.tiff

- Additionalfile1.pdf

- HaydetalTables.xlsx

- Additionalfile2.pdf 